\title{
Search for Axions from Nuclear Power Reactor with a High-purity Germanium Detector
}

\author{
H. M. Chang and H. T. Wong* \\ (on behalf of the TEXONO Collaboration) \\ Institute of Physics, Academia Sinica, Taipei 11529, Taiwan \\ E-mail: chmephys.sinica.edu.tw, htwong@phys.sinica.edu.tw
}

\begin{abstract}
This article reports the first study of possible emissions of axions from power reactors using Primakoff and Compton conversions as the detection mechanisms. The experiment was performed at the Kuo-Sheng (KS) Nuclear Power Station with a high-purity germanium detector of mass 1.06 $\mathrm{kg}$ at a distance of $28 \mathrm{~m}$ from the $2.9 \mathrm{GW}$ reactor core. The expected experimental signatures are mono-energetic lines produced by Primakoff or Compton conversions of axions at the detector. Based on 459.0/96.3 days of reactor ON/OFF data, no evidence of axion emission was observed and constraints on axion couplings $g_{a \gamma \gamma}$ and $g_{a e e}$ versus axion mass $m_{a}$ within the framework of invisible axion models were placed. The KSVZ and DFSZ models can be excluded for $10^{4} \mathrm{eV}$ $\lesssim m_{a} \lesssim 10^{6} \mathrm{eV}$. Model-independent constrains on $g_{a \gamma \gamma} \cdot g_{a N N}^{1}<7.7 \times 10^{-9} \mathrm{GeV}^{-2}$ for $m_{a} \lesssim 10^{5}$ $\mathrm{eV}$ and $g_{a e e} \cdot g_{a N N}^{1}<1.3 \times 10^{-10}$ for $m_{a} \lesssim 10^{6} \mathrm{eV}$ at $90 \%$ confidence level were derived. This experimental approach provides a unique probe for axion mass at the $\mathrm{keV}-\mathrm{MeV}$ range not accessible to the other techniques.
\end{abstract}

Identification of dark matter 2008

August 18-22, 2008

Stockholm, Sweden

${ }^{*}$ Poster Presented at IDM08. 


\begin{tabular}{llllll}
\hline \hline Channel & $\begin{array}{l}E_{\gamma} \\
(\mathrm{keV})\end{array}$ & Mode & $\begin{array}{l}\Phi_{\gamma} \\
\left(10^{10} \mathrm{~cm}^{-1} \mathrm{~s}^{-1}\right)\end{array}$ & $\begin{array}{l}\text { ON-OFF } \\
\left(\mathrm{day}^{-1} \mathrm{~kg}^{-1}\right)\end{array}$ & $\begin{array}{l}\mathrm{S}_{u} \\
\left(\mathrm{day}^{-1} \mathrm{~kg}^{-1}\right)\end{array}$ \\
\hline $\mathrm{p}(\mathrm{n}, \gamma / \mathrm{a}) \mathrm{d}$ & 2230 & Isovector M1 & 22.1 & $-0.04 \pm 0.03$ & $<0.02$ \\
${ }^{10} \mathrm{~B}(\mathrm{n}, \alpha)^{7} \mathrm{Li}^{*}$ & 478 & $\mathrm{M} 1\left(\frac{1}{2}^{-}\right) \rightarrow\left(\frac{3}{2}^{-}\right)$ & 24.7 & $-0.09 \pm 0.36$ & $<0.49$ \\
${ }^{91} \mathrm{Y}^{*}$ & 555 & $\mathrm{M} 4\left(\frac{9}{2}^{+}\right) \rightarrow\left(\frac{1}{2}^{-}\right)$ & 2.10 & $-0.34 \pm 0.15$ & $<0.05$ \\
${ }^{97} \mathrm{Nb}^{*}$ & 743 & $\mathrm{M} 4\left(\frac{1}{2}^{-}\right) \rightarrow\left(\frac{9}{2}^{+}\right)$ & 4.81 & $0.19 \pm 0.31$ & $<0.69$ \\
${ }^{135} \mathrm{Xe}^{*}$ & 526 & $\mathrm{M} 4\left(\frac{11}{2}^{-}\right) \rightarrow\left(\frac{3}{2}^{+}\right)$ & 0.85 & $0.37 \pm 0.15$ & $<0.62$ \\
${ }^{137} \mathrm{Ba}^{*}$ & 662 & $\mathrm{M} 4\left(\frac{11}{2}^{-}\right) \rightarrow\left(\frac{3}{2}^{+}\right)$ & 0.37 & $-0.19 \pm 0.39$ & $<0.46$ \\
\hline \hline
\end{tabular}

Table 1: Summary of magnetic transitions and their estimated fluxes from reactor. The measured reactor ON-OFF event rates and the $90 \% \mathrm{CL}$ upper limits $\left(\mathrm{S}_{u}\right)$ are also listed.

This axion research [1] is a by-product of the research program on neutrino magnetic moment [2] pursued by the TEXONO Collaboration. Axions can be emitted in competition with the photons as a result of neutron captures or nuclear de-excitations at reactor. There are six prominent channels of magnetic gamma-transitions at typical nuclear reactors, as listed in Tab. 11. Thermal neutron captures on the ${ }^{10} \mathrm{~B}$ in the control rods and on proton in the cooling water produce $\alpha+{ }^{7} \mathrm{Li}^{*}$ and $\mathrm{d}+\gamma / a$, respectively. The sources of ${ }^{91} \mathrm{Y}^{*},{ }^{97} \mathrm{Nb}^{*},{ }^{135} \mathrm{Xe}^{*}$, and ${ }^{137} \mathrm{Ba}^{*}$ are all fission daughters, and their de-excitations as well as ${ }^{7} \mathrm{Li}^{*}$ 's may emit axions. The axion flux $\left(\phi_{a}\right)$ at a distance $L$ from a reactor core of fission rate $R_{f}$ can be described by

$$
\phi_{a}(L)=\frac{R_{f} \cdot \Phi_{\gamma}}{4 \pi L^{2}} \cdot \frac{\Gamma_{a}}{\Gamma_{\gamma}} \cdot P_{d k} \cdot P_{i n t},
$$

where $\left(\Gamma_{a} / \Gamma_{\gamma}\right)$ is the branching ratio of axion emissions in the transitions. $P_{d k}$ and $P_{i n t}$ are the probabilities of the axions surviving the flight from reactor core to detector without decays or interactions.

The search strategies for reactor axions were inspired by a previous experiment [3]. Two interaction mechanisms of axions with matter were studied: Primakoff and Compton conversions. These processes are respectively and independently sensitive to $g_{a \gamma \gamma}$ and $g_{a e e}$. The total energy of the axions can be fully converted by either of the two processes into measurable ionization energy in the HPGe, such that the experimental signatures are the presence of mono-energetic lines at the known axion energies $\left(E_{a}\right.$ 's) during the Reactor ON periods. Evidence of reactor axions would manifest as peaks at the known energies of Tab. 11 in the Reactor ON-OFF residual spectra. As illustrations, the ON/OFF background spectra and the ON-OFF residual spectra for the six candidate lines are depicted in Figs. 1 and 2 , respectively. The count rates and their errors of the various transitions shown in Tab. 11 were derived by best-fits of the residual spectra to Gaussian lines at fixed $E_{a}$ 's and resolutions. No excess were observed in all channels and upper limits of the signal rates $\left(S_{u}\right)$ at $90 \%$ confidence level (CL) were derived and listed in Tab. 1. The experimentally measured upper limits $S_{u}$ of Tab. 1 1 can be translated to bounds among the axion parameters: $m_{a}$, $g_{a \gamma \gamma}, g_{a e e}, g_{a N N}^{0}$ and $g_{a N N}^{1}$. Two approaches were adopted [四]: (a) specific models were used and tested, and (b) model-independent constraints among the parameters were derived.

The model-dependent exclusion plots of $g_{a \gamma \gamma}$ and $g_{a e e}$ versus $m_{a}$ are depicted in Figs. 3 (a) 


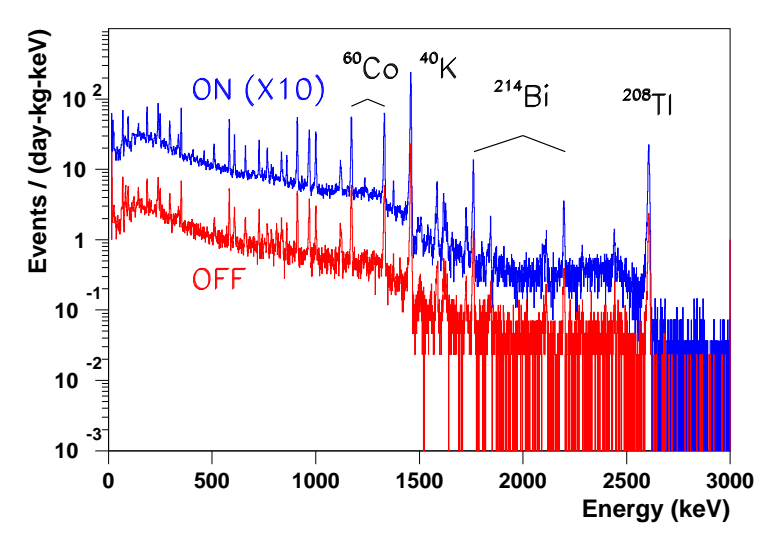

Figure 1: The reactor ON and OFF spectra of HPGe detector.

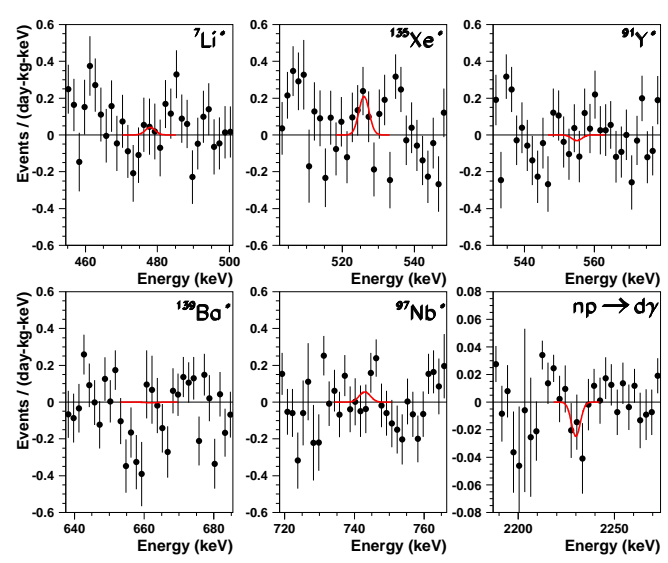

Figure 2: The residual spectra for the six specific channels, with the best-fit Gaussian peaks overlaid.

and $3(\mathrm{~b})$. The model-dependence was introduced by fixing $\left(\Gamma_{a} / \Gamma_{\gamma}\right)_{p n}$ as a function of $m_{a}$ [1]. The boundaries defined by "Int", "Decay" and "Kine" are constraints due to axions interactions with matter, decays in flights and kinematics, respectively. The bounds from TEXONO improve on those of Ref. [3] marked "Zn" in Figs. 3(a) and 3(b) by two orders of magnitude, owing to enhanced axion flux, lower background and larger data sample. The TEXONO results define the global exclusion boundaries [1] in $g_{a \gamma \gamma}$ for $10^{3} \mathrm{eV} \lesssim m_{a} \lesssim 10^{6} \mathrm{eV}$ and in $g_{a e e}$ for $10^{2} \mathrm{eV} \lesssim m_{a} \lesssim$ $10^{6} \mathrm{eV}$. Model-independent constrains on $g_{a \gamma \gamma}$ and $g_{a e e}$ as functions of $g_{a N N}^{1}$ and $\left(\Gamma_{a} / \Gamma_{\gamma}\right)_{p n}$ are

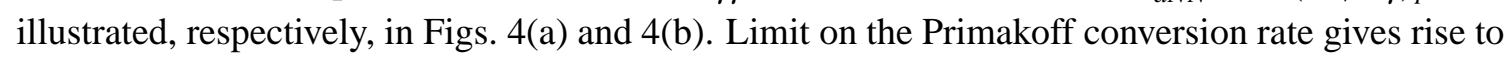
the constraint

$$
g_{a \gamma \gamma}^{2} \cdot\left(\frac{\Gamma_{a}}{\Gamma_{\gamma}}\right)_{p n}<5.9 \times 10^{-17} \mathrm{GeV}^{-2} \Rightarrow g_{a \gamma \gamma} \cdot g_{a N N}^{1}<7.7 \times 10^{-9} \mathrm{GeV}^{-1}
$$

which are applicable within the ranges of $m_{a} \lesssim 10^{5} \mathrm{eV}$ and $g_{a \gamma \gamma} \lesssim 20 \mathrm{GeV}^{-1}$, bounded by kinematics and axion interactions in flight, respectively. Similarly, the limit on the Compton conversion rate leads to

$$
g_{a e e}^{2} \cdot\left(\frac{\Gamma_{a}}{\Gamma_{\gamma}}\right)_{p n}<1.7 \times 10^{-20} \Rightarrow g_{a e e} \cdot g_{a N N}^{1}<1.3 \times 10^{-10}
$$

for $m_{a} \lesssim 10^{6} \mathrm{eV}$ and $g_{a e e} \lesssim 0.2$.

\section{References}

[1] H. M. Chang et al., Search for axions from the Kuo-Sheng nuclear power reactor with a high-purity germanium detector, Phys. Rev. D 75052004 (2007), and references therein.

[2] H. B. Li et al., Limit on the Electron Neutrino Magnetic Moment from the Kuo-Sheng Reactor Neutrino Experiment, Phys. Rev. Lett. 90131802 (2003).

H. T. Wong et al., Search of neutrino magnetic moments with a high-purity germanium detector at the Kuo-Sheng nuclear power station, Phys. Rev. D 75012001 (2007).

[3] F. T. Avignone III et al., Search for axions from the 1115-keV transition of ${ }^{65}$ Cu, Phys. Rev. D 37618 (1988). 


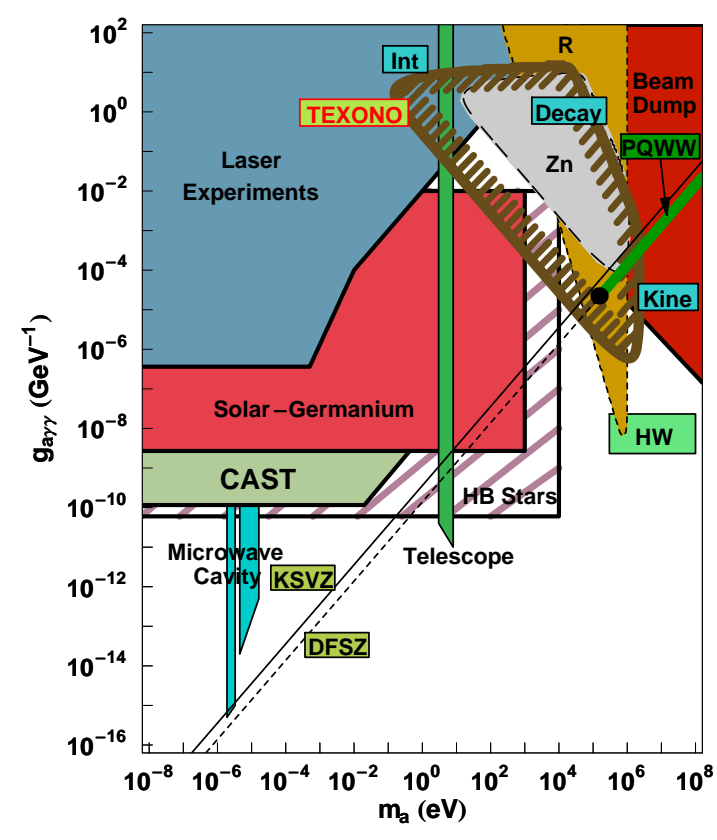

(a)

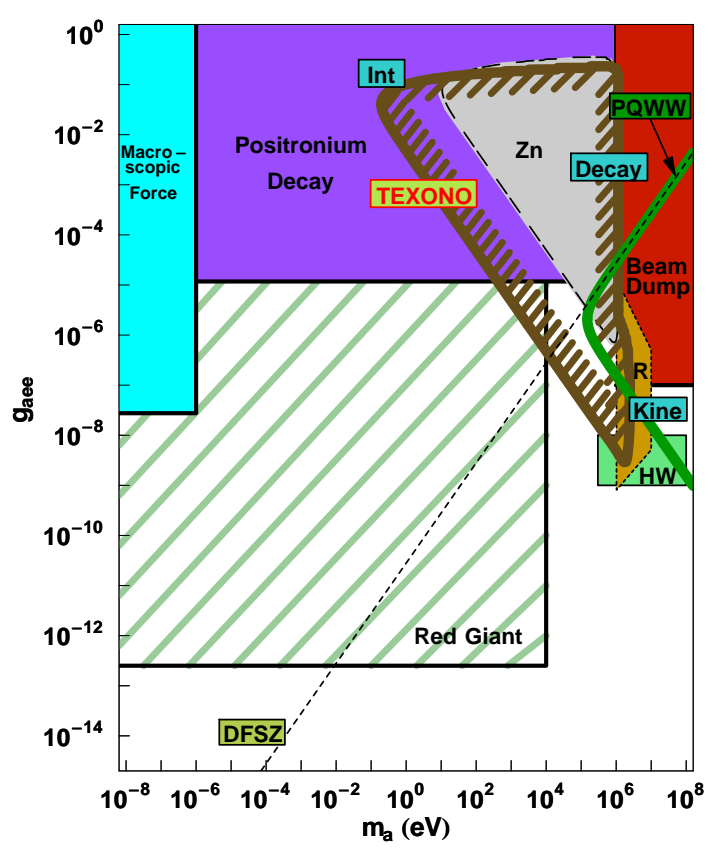

(b)

Figure 3: Exclusion plots of (a) $g_{a \gamma \gamma}$ versus $m_{a}$ for $g_{a e e}=0$ and (b) $g_{a e e}$ versus $m_{a}$ for $g_{a \gamma \gamma}=0$. Our modeldependent limits are denoted by "TEXONO".

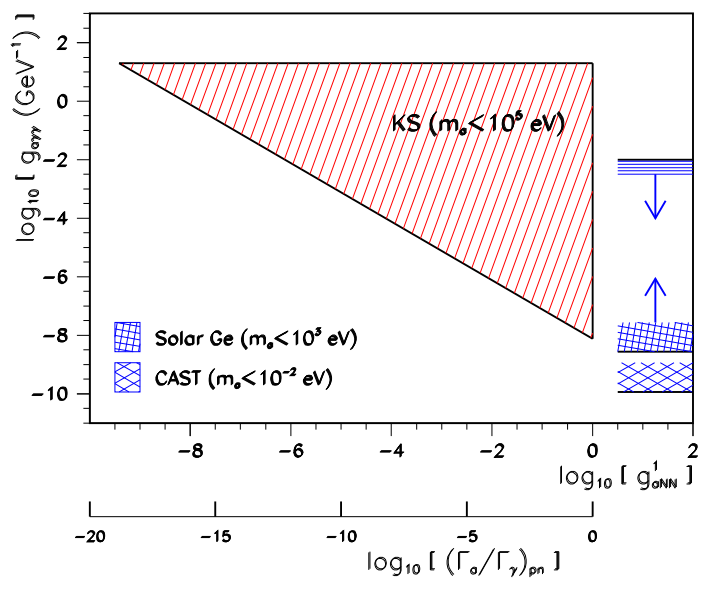

(a)

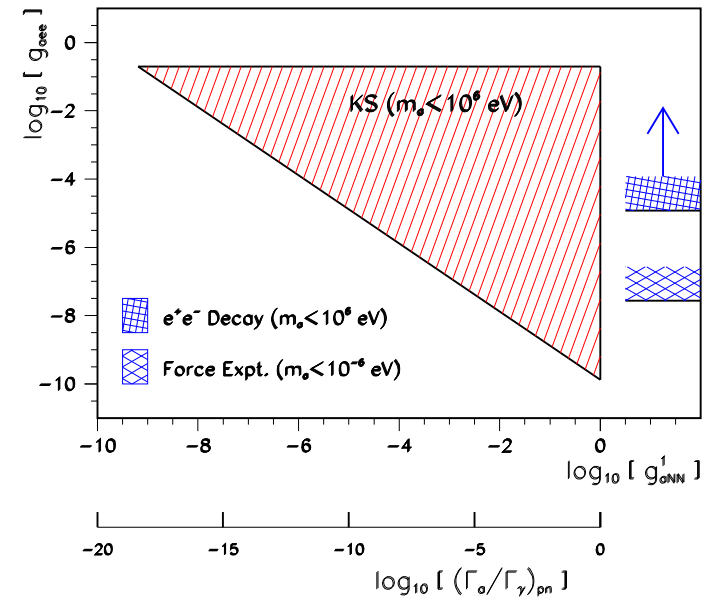

(b)

Figure 4: The model-independent exclusion region of TEXONO axion search experiment labeled by "KS" for (a) $g_{a \gamma \gamma}$ and (b) $g_{a e e}$ on the $\left(\Gamma_{a} / \Gamma_{\gamma}\right)_{p n}$ and $g_{a N N}^{1}$ axes. The $g_{a \gamma \gamma}$ and $g_{a e e}$ limits from the leading laboratory experiments are also shown for comparisons. The range of validity in $m_{a}$ are indicated. 